\title{
Regulación y competencia en el sector de la cultura en Andalucía
}

\section{Regulation and competition in the culture sector in Andalusia}

\author{
Luis Palma Martos \\ María Luisa Palma \\ Aarón Espinosa Espinosa \\ Universidad de Sevilla
}

José Félix Riscos

Agencia de Defensa de la Competencia de Andalucía

\section{Resumen}

El artículo evalúa el seguimiento de los principios de buena regulación y competencia en el sector cultural andaluz, mediante el análisis de las actuaciones de la Agencia de Defensa de la Competencia de Andalucía, recogidas en sus recomendaciones a proyectos y anteproyectos normativos de la Administración Pública, entre 2008 y 2018. Los resultados muestran que los informes sobre el sector cultural comienzan en 2016 y se concentran en tres sectores: audiovisual, patrimonio y artes escénicas. Asimismo, muestran un alto nivel de seguimiento de las recomendaciones en la normativa. El artículo aporta un marco analítico y metodológico extensible a otros territorios para evaluar el papel de los órganos de defensa de la competencia en la mejora de la regulación y funcionamiento de los mercados en el sector cultural.

Palabras clave: políticas de competencia, regulación, sector cultural, Andalucía.

Clasificación JEL: H11, L51, Z18.

\begin{abstract}
The aim of this article is to evaluate the monitoring of the principles of regulation and competition in the Andalusian cultural sector, by analyzing the actions of the Agency for the Defense of Competition of Andalusia, included in its recommendations for projects and draft bill regulations of the Public Administration, between 2008 and 2018. The results show that reports on the cultural sector begin in 2016 and focus on three sectors: audiovisual, heritage and performing arts. They also show a high level of follow-up of the recommendations in the regulations. The article provides an analytical and methodological framework extensible to other territories to evaluate the role of competition bodies in improving the regulation and functioning of markets in the cultural sector.
\end{abstract}

Keywords: competition policy, regulation, cultural sector, Andalusia.

JEL classification: H11, L51, Z18. 


\section{Introducción}

La regulación de la actividad económica representa una de las distintas formas de intervención de las Administraciones públicas (AAPP) que, en países como España, se desenvuelven en distintos niveles de gobierno con capacidad normativa. Una buena regulación económica salvaguarda intereses sociales legítimos al distorsionar lo menos posible los incentivos y el libre juego del mercado, fomenta la competitividad empresarial al reducir los costes operativos, y suprime trabas y barreras innecesarias o desproporcionadas a las actividades productivas. La regulación eficiente también contribuye a la ampliación de la capacidad de elección de los ciudadanos en los mercados (OCDE, 2014).

Sin embargo, la calidad reguladora -un componente fundamental de la calidad institucional- representa una de las debilidades de la economía española. Cuando en un contexto mundial se evalúa el nivel de desempeño institucional que correspondería a su capacidad productiva, el país se ubica un $26 \%$ por debajo de su potencial, lo que lleva a revisar aspectos tales como el exceso de regulación y sus costes para las empresas, la facilidad para emprender negocios, controles de precios y barreras a la libre competencia (Alcalá y Jiménez, 2018).

Este rezago de la economía nacional muestra la pertinencia de evaluar los mecanismos de regulación en materia de competencia, unidad de mercado y actividades económicas, y en particular, de las normas con rango de ley y las disposiciones reglamentarias emitidas por las AAPP españolas. A este nivel, los órganos de promoción y defensa de la competencia autonómicas juegan un papel determinante (Román y Riscos, 2019).

En este contexto, el presente trabajo tiene un doble propósito: el primero, evaluar el grado de seguimiento, por parte de los centros directivos en el área de política cultural de la Junta de Andalucía, de las recomendaciones, recogidas en forma de dictamen, en los informes sobre proyectos normativos aprobados por el Consejo de Defensa de la Competencia (CDC) de la Agencia de Defensa de la Competencia de Andalucía (ADCA). Segundo, observar la incidencia que han tenido en el ámbito de la mejora de la regulación, especialmente en el sector cultural, dos iniciativas normativas: el Decreto-ley 5/2014 de 22 de abril, de medidas normativas para reducir las trabas administrativas para las empresas (BOJA N. ${ }^{\circ} 82 / 2014$, p. 7-51), que amplía las funciones de la ADCA en esta materia, y la Resolución de 19 de abril de 2016 de la Agencia de Defensa de la Competencia de Andalucía, del Consejo de Defensa de la Competencia de Andalucía, por la que se aprueban los criterios para determinar la incidencia de un proyecto normativo en la competencia efectiva, la unidad de mercado y las actividades económicas (BOJA N. . 90/2016, p. 8-10) . Con ello pretendemos contrastar si la mayor proliferación de informes, recomendaciones e inclusión de las mismas en los proyectos normativos, coinciden con una fecha posterior a la publicación de la normativa anteriormente citada, además de seguir los principios de la buena regulación. El periodo de estudio es el comprendido entre los años 2008 (año de entrada en funcionamiento de la ADCA) y 2018. 
A fin de alcanzar estos objetivos, se realiza un análisis comparativo entre los textos inicialmente elaborados por el centro directivo proponente, las recomendaciones realizadas en los informes aprobados por el CDC a cada propuesta de norma, y los textos normativos finalmente publicados en el Boletín Oficial de la Junta de Andalucía (BOJA). En el periodo considerado, el CDC ha aprobado un total de 153 informes de proyectos normativos, de los cuales 12 se refieren al sector cultural, lo que supone un $7,8 \%$ del total. Es preciso señalar que el primer informe referido al sector cultural data de 2016, tras la aprobación de las iniciativas normativas.

El análisis identifica, a partir de un exhaustivo examen de los informes del CDC, los principales problemas que los proyectos de normas incorporan desde la perspectiva de la mejora de la regulación, la competencia efectiva y la unidad de mercado. Así mismo, evalúa el porcentaje y la naturaleza de las recomendaciones atendidas.

El trabajo contribuye a la literatura sobre regulación y competencia en el sector cultural de Andalucía, materia que no ha sido abordada hasta el momento, en dos aspectos. El primero, identifica las principales restricciones que los proyectos normativos que regulan el sector cultural suelen incorporar desde la perspectiva de la competencia efectiva y la regulación eficiente. Estas restricciones son extrapolables a otros territorios y ámbitos de análisis. El segundo, permite observar las buenas prácticas aceptadas por los centros directivos a partir de las recomendaciones realizadas por el CDC, lo que implica, no solo reforzar el diálogo entre instituciones públicas con objetivos diversos, sino también alcanzar con sus iniciativas objetivos de interés general que pueden resumirse en una mejor asignación de los recursos públicos y la posibilidad de que una buena regulación contribuya a dinamizar un sector clave para la economía de Andalucía.

El trabajo se organiza de la siguiente manera: después de esta introducción, se realiza una revisión de la literatura sobre política de competencia y las razones para una regulación económica eficiente; en la tercera parte se recoge la metodología utilizada por la ADCA en la evaluación de los informes normativos; en la cuarta se presentan los principales resultados del análisis y, por último, en la quinta sección, se finaliza con un apartado de conclusiones.

\section{Revisión de la literatura}

La teoría económica, la experiencia histórica y la evidencia empírica permiten establecer los efectos positivos de las políticas de competencia para incentivar la capacidad emprendedora y la inversión, aumentar las actividades de innovación, mejorar el comportamiento de los precios y, en definitiva, conseguir una mayor competitividad y productividad de la economía en su conjunto.

En general, las economías más desarrolladas son aquellas que han logrado crear un sistema de regulación que facilita las interacciones en el mercado y protege los intereses generales sin obstaculizar de forma innecesaria la actividad económica y la inversión productiva. Así mismo, los países donde existe una competencia efectiva 
tienden a registrar menores niveles de desempleo, inflación y pobreza, al tiempo que sus habitantes gozan de mayor calidad de vida y bienestar.

La libre competencia incentiva la capacidad emprendedora y la dinámica empresarial a través de mecanismos como la predisposición a innovar, la mejora de la organización, la adecuación de la estructura de costes y el aumento de la productividad. El efecto más notorio de este aumento de la competencia son la existencia de menores precios a los consumidores, y un aumento de la cantidad, calidad y variedad de productos, con todo lo cual se contribuye al crecimiento económico y a mejorar el bienestar de países y regiones (OCDE, 2014).

En esencia, los pilares de la economía social de mercado lo constituyen la libre competencia y la regulación económica eficiente. Estos pilares operan por dos vías: en primer lugar, a través de la aplicación de leyes que garanticen la competencia efectiva y eviten los cárteles y el abuso del poder de mercado; y en segundo, mediante la regulación eficiente de los mercados, asumiendo de forma técnica y rigurosa la tarea de resolver sus fallos, así como mediante la reducción de trabas desproporcionadas, la eliminación de barreras innecesarias y la simplificación de procedimientos burocráticos (Gráfico 1).

\section{GRÁFICO 1}

\section{RELACIONES ENTRE DEFENSA DE LA COMPETENCIA, CRECIMIENTO ECONÓMICO Y BIENESTAR}

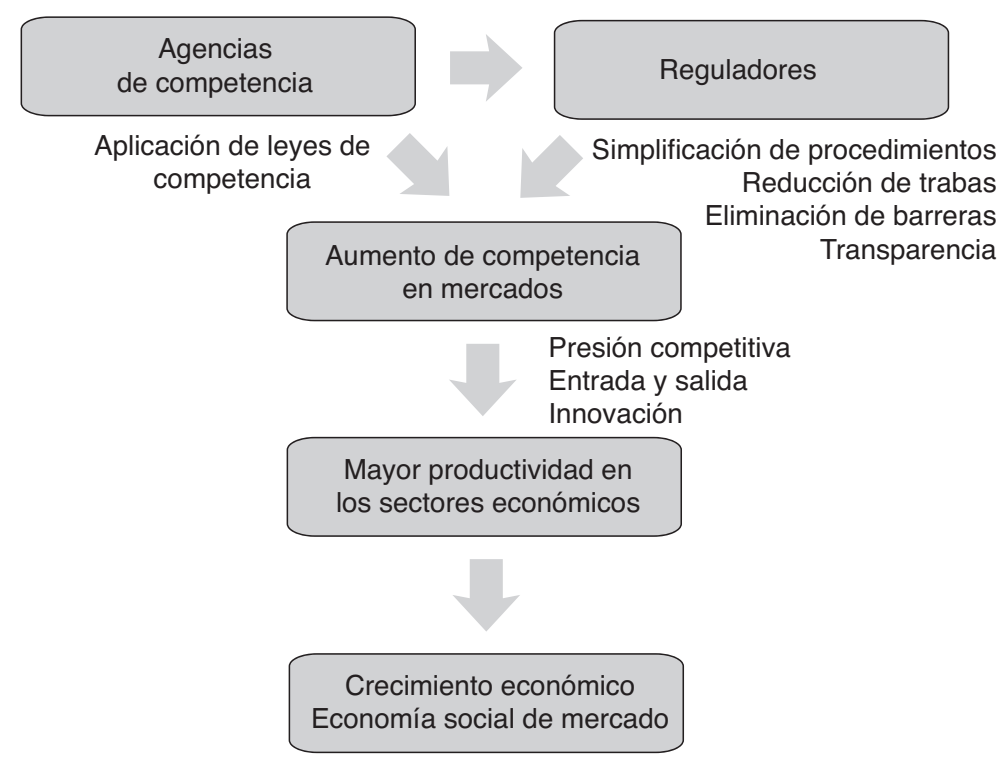

FUENTE: Elaboración propia con base en OCDE (2014). 
En este contexto, cabe señalar que existe una relación profunda entre las políticas de competencia, especialmente entre la promoción de la competencia, y la mejora de la regulación económica. La labor de promoción de la competencia hace referencia a todas aquellas actuaciones orientadas al fomento de un entorno competitivo para el desarrollo de las actividades económicas mediante el empleo de mecanismos no coactivos.

Para Alcalá y Jiménez (2018), «La consecución de una alta productividad y el sostenimiento a largo plazo de su crecimiento son objetivos difícilmente alcanzables sin normas competitivas adecuadas y sin organismos reguladores con elevado nivel profesional y con independencia del poder político y de los grandes grupos económicos y de interés» (p. 17).

Uno de los instrumentos más destacados de la promoción de la competencia lo constituye el control de los proyectos normativos, máxime si se tiene en cuenta que, en ocasiones, la propia actuación de los poderes públicos introduce restricciones o trabas innecesarias y desproporcionadas al acceso o ejercicio de las actividades económicas, lo cual afecta o distorsiona el libre juego competitivo de los operadores económicos. El análisis y supresión de estas restricciones o trabas es el nexo que relaciona la promoción de la competencia y la mejora de la regulación económica.

Las políticas de promoción de la competencia y de mejora de la regulación económica también se justifican en el ámbito de la coordinación de políticas autonómicas. A este nivel, se requiere de manera permanente evaluar la incidencia, en materia de competencia y unidad de mercado, de las normas con rango de ley y las disposiciones reglamentarias elaboradas por las AAPP.

La literatura empírica que respalda las políticas a favor de la competencia es abundante en el entorno europeo y valida gran parte de las conexiones teóricas ya planteadas. En el estudio clásico de Nikell (1996), en el que se examinan alrededor de 672 compañías del Reino Unido, se plantea que «[...] la competencia, medida por un mayor número de competidores o por niveles más bajos de alquileres, se asocia con una tasa significativamente mayor de crecimiento de la productividad total de los factores» (p. 741). En cuanto a los resultados de su estudio, Nikell halla evidencia según la cual, entre 1975 y 1986 las empresas más competitivas fueron más productivas (crecieron 3,8\%-4,6\% más) que las menos competitivas.

Otro estudio, el realizado por Clougherty (2010), examina el entorno transnacional de la política de competencia. Este autor analizó tanto los recursos destinados a esta política como la carga de trabajo en 32 casos de jurisdicciones antimonopolio en el periodo comprendido entre 1992 y 2007 , y halló que un aumento de los fondos de la política de competencia, de alrededor de 60 millones de dólares al año, supondría un aumento del crecimiento económico -medido a través del PIB per cápita- del $0,84 \%$. Una faceta destacable de este trabajo es la importancia de apoyar las políticas de competencia mediante fondos públicos, lo que permite validar la actividad de defensa de la competencia en Andalucía.

Desde el punto de vista metodológico, Kronthaler (2010) proporciona uno de los primeros estudios que utilizan un enfoque empírico basado en datos de panel para 
explicar la efectividad de las políticas antimonopolio. En su estudio estima una tendencia temporal que relaciona el desempeño de un conjunto de factores con leyes de competencia recién promulgadas.

Las bondades de la competencia efectiva también se observan en el funcionamiento de los mercados laborales. Fiori et al. (2012) muestran para un panel de datos de los países de la OCDE, que durante 22 años (1980-2002) las interacciones entre políticas e instituciones en los mercados de productos y laborales son claves para aumentar la tasa de empleo a largo plazo. Del estudio se establece que la desregulación del mercado de productos es más efectiva cuando la regulación del mercado laboral es alta.

Por su parte, Buccirossi et al. (2013) muestran que la aplicación de la política de competencia favorece el aumento de la inversión y la productividad. Según los resultados de este estudio, las mejoras en política de competencia en 12 países de la OCDE explican cerca de una quinta parte de los aumentos en productividad en 22 industrias. El impacto positivo de las políticas analizadas se extiende para el periodo 1995-2005, y comprende la adecuada configuración institucional y el ejercicio de actividades antimonopolio, así como los sistemas legales que procuren las complementariedades entre política de competencia y la eficiencia de la aplicación de la ley.

En 2013, Petersen publica un estudio sobre las consecuencias macroeconómicas de las leyes de competencia. Este autor analiza el efecto a corto plazo de la introducción de una ley de competencia sobre el ingreso per cápita y las tasas de crecimiento en una amplia muestra de países (Petersen, 2013).

Por último, Gutmann y Voigt (2014) muestran que políticas de competencia eficientes generan aumentos del PIB per cápita de 2-3\% anual en una amplia muestra de 105 países que analizaron durante 40 años. Estos autores, que cuestionan la validez de los resultados de Clougherty por problemas de endogeneidad, subrayan la utilidad de las políticas de competencia en procesos de convergencia. Para Gutmann y Voigt, en países de bajos ingresos la regulación a favor de la competencia aumenta los niveles de inversión y puede, consecuentemente, aumentar el crecimiento y promover la reducción de las disparidades económicas y sociales entre regiones. Según concluye su estudio, una ganancia adicional de la política de competencia es el descenso de la corrupción después de que se implantan las leyes en esta materia.

En definitiva, los estudios referenciados aportan evidencias sobre los efectos positivos de la aplicación de las políticas de competencia, tanto por su impacto sobre la productividad y nivel de vida de países y regiones, como por su incidencia en la mejora del desempeño de otros mercados, como el de bienes y servicios, y el laboral.

\section{Metodología}

Como se ha expuesto, la regulación de las actividades económicas incide sobre el funcionamiento de los mercados y establece el marco competitivo de las empresas. La experiencia y la teoría de la regulación apuntan al uso de la regulación econó- 
mica como una herramienta que, por un lado, contribuye a incrementar el bienestar social cuando resuelven los fallos del mercado pero, en caso contrario, puede generar costes y distorsiones en la asignación de los recursos. Por tanto, la calidad de la regulación económica es un elemento clave para determinar el buen funcionamiento de las actividades económicas.

\subsection{Instrumentos de mejora de la regulación en Andalucía: el informe de normas}

En Andalucía, un factor clave ha sido concentrar las competencias y actuaciones para la mejora de la regulación económica en un organismo especializado. En concreto, en la ADCA, con la aprobación de la Ley 3/2014, de 1 de octubre, de medidas normativas de reducción de trabas administrativas para las empresas en Andalucía; ello supuso una considerable apuesta por una agencia independiente, que ya contaba con las funciones de promoción y defensa de la competencia de los mercados. Con dicha Ley 3/2014, se modificó la Ley 6/2007, de 26 de junio, de Promoción y Defensa de la Competencia de Andalucía, optando por un modelo de autoridad de competencia que refuerza las tareas de asesoramiento a la Administración de la Junta de Andalucía, al establecer entre los fines de la ADCA en su artículo 2, «contribuir a mejorar la regulación económica».

Por otra parte, la ADCA, de conformidad con el artículo 3.i de dicha Ley 6/2007, de 26 de junio, asumió entre sus funciones la de «informar los anteproyectos de ley y proyectos de reglamento de la Administración de la Junta de Andalucía que incidan sobre las actividades económicas, afecten a la competencia efectiva o la unidad de mercado, de forma que se garanticen los intereses generales».

Esta labor consultiva es un instrumento preciso que ayuda a mejorar la calidad reguladora, que se ha desarrollado mediante la Resolución de 19 de abril de 2016 del CDC. Por esta norma, se aprueban criterios para determinar la incidencia de un proyecto normativo en la competencia efectiva, unidad de mercado y actividades económicas. Para determinar el impacto de una norma y elaborar el informe normativo, se sigue una serie de fases diferenciadas e interrelacionadas de análisis que incluyen los siguientes aspectos:

\section{a) Identificación de los principios de la buena regulación económica}

En esta fase se debe partir de que los poderes públicos, al elaborar o aplicar las normas que inciden en las actividades económicas, actuarán según los llamados principios de buena regulación. Esto es, los principios de eficiencia, necesidad, proporcionalidad, seguridad jurídica, transparencia, accesibilidad, simplicidad y eficacia (Cuadro 1). 


\section{CUADRO 1}

\section{LOS PRINCIPIOS DE LA BUENA REGULACIÓN ECONÓMICA Y LAS PREGUNTAS QUE RESUELVE}

\begin{tabular}{|c|c|}
\hline Principio & Preguntas \\
\hline Eficiencia & $\begin{array}{l}\text { - ¿Superan los beneficios de la medida a sus costes? Equilibrio razonable } \\
\text { entre la ventaja que la medida comporta para la finalidad perseguida y } \\
\text { la eventual restricción que origina. }\end{array}$ \\
\hline Necesidad & $\begin{array}{l}\text { - ¿Cuáles son los objetivos y finalidades perseguidos? } \\
\text { - La norma se justifica en una razón de interés general o responde a un } \\
\text { fallo de mercado. }\end{array}$ \\
\hline Proporcionalidad & $\begin{array}{l}\text { - ¿El objetivo que se persigue podría quedar igualmente salvaguardado } \\
\text { con medidas o instrumentos menos restrictivos } \\
\text { ¿Constituye la norma la mejor alternativa? } \\
\text { - ¿Es, además, la menos restrictiva de entre las que podrían satisfacer la } \\
\text { finalidad pretendida? }\end{array}$ \\
\hline Seguridad jurídica & $\begin{array}{l}\text { - ¿El nuevo marco normativo será estable y predecible, con certidumbre } \\
\text { para ciudadanos y empresas? } \\
\text { ¿Se creará un marco normativo sencillo, claro y poco disperso? } \\
\text { - ¿La iniciativa normativa es coherente con el resto del ordenamiento } \\
\text { jurídico, nacional y de la UE? }\end{array}$ \\
\hline Transparencia & $\begin{array}{l}\text { - ¿Los objetivos de la norma se definen claramente? } \\
\text { - ¿Estos fines se justifican en el preámbulo o en la exposición de moti- } \\
\text { vos? } \\
\text { - ¿Los documentos propios de su proceso de elaboración son públicos? }\end{array}$ \\
\hline Accesibilidad & $\begin{array}{l}\text { - ¿Se establecen mecanismos de consulta y de participación activa de los } \\
\text { potenciales destinatarios? }\end{array}$ \\
\hline Simplicidad & $\begin{array}{l}\text { - ¿El marco regulatorio es sencillo, claro y poco disperso, y facilita el } \\
\text { conocimiento y la comprensión del mismo? }\end{array}$ \\
\hline Eficacia & $\begin{array}{l}\text { - ¿Se trata de una medida congruente y adecuada para hacer frente al } \\
\text { fallo de mercado o para alcanzar el objetivo de interés general? } \\
\text { ¿Cómo contribuye a la consecución de la finalidad perseguida? } \\
\text { (relación de causalidad). }\end{array}$ \\
\hline
\end{tabular}
(2017).

FUENTE: Elaboración propia con base en la Guía para la mejora de la regulación económica en Andalucía

\section{b) Efectos sobre la competencia efectiva}

Esta fase tiene por objeto evitar que la normativa y la regulación introduzcan restricciones en el comportamiento competitivo de los operadores económicos, establezcan obstáculos o barreras al mantenimiento de una competencia efectiva en los mercados, o alteren los incentivos existentes. El objetivo es, por tanto, que la regulación no restrinja el comportamiento de los distintos agentes económicos, por lo que hay que contar si la norma introduce alguna limitación en el libre acceso de las empresas al mercado, restringe la competencia entre las empresas que operan en el mercado o reduce los incentivos para competir. 


\section{c) Efectos sobre la unidad de mercado}

La Ley 20/2013, de 9 de diciembre, de Garantía de la Unidad de Mercado (LGUM), establece principios y normas básicas para la mejora de la regulación. Según esta ley, la unidad de mercado se fundamenta en la libre circulación y establecimiento de los operadores económicos, en la libre circulación de bienes y servicios por todo el territorio nacional sin que ninguna autoridad pueda obstaculizarla directa o indirectamente, y en la igualdad de las condiciones básicas de ejercicio de la actividad económica. Su objetivo se orienta a eliminar barreras administrativas y trabas burocráticas, y a establecer un marco regulador eficiente para las actividades económicas a través de una serie de técnicas de distinto contenido y alcance.

\section{d) Impacto sobre las actividades económicas}

En esta fase se evalúa si el proyecto incide sobre las actividades económicas, en particular, sobre las empresas y las pymes, e igualmente sobre el empleo, los consumidores y usuarios, y los precios de los bienes y servicios.

\subsection{Referentes e instrumentos de seguimiento y evaluación}

Para definir la mejora de la regulación, se parte de la noción planteada en el Informe Mandelkern, según el cual este tipo de regulación se caracteriza porque: 1) «Busca mejorar y simplificar el entorno regulador»; 2) Debe usarse «solo cuando sea necesaria»; 3) «Debe ser apropiada y proporcionada con su objetivo»; y 4) «Debería ser transparente y accesible para todos y tan simple como sea posible» (Mandelkern Group, 2001).

Como se ha expuesto, el objetivo de este trabajo es evaluar el seguimiento de los principios de buena regulación y competencia mediante el análisis de la incorporación a la normativa finalmente publicada por la Administración pública de las recomendaciones realizadas a los proyectos y anteproyectos normativos por parte de la ADCA, desde su entrada en funcionamiento en 2008 hasta la actualidad. Para ello, se realiza una evaluación exhaustiva de todos los informes normativos realizados por la ADCA relativos al sector cultural, destacando los principales subsectores o ámbitos objeto de regulación, los principales temas abordados en cada uno de ellos (principios de buena regulación y competencia afectados) y cuántos son incorporados a los proyectos o anteproyectos normativos.

El análisis realizado se basa en los principios de la buena regulación afectados, que fundamentan las recomendaciones efectuadas al articulado de los proyectos de normas. A continuación, se pretende cumplir con el segundo objetivo del trabajo, contrastando no solo cuáles de los principios de buena regulación más afectados en estas recomendaciones han sido aceptados e incluidos en la redacción del articulado concreto de la normativa finalmente publicada, sino en qué periodo temporal se producen, tanto una mayor proliferación de informes como la inclusión de recomendaciones. Como ya hemos indicado con anterioridad, el primer informe sobre 
el sector objeto de estudio está fechado en 2016, es decir, tras la promulgación de las iniciativas de 2014 y 2016 antes citadas. De esta forma, se determina el grado de seguimiento de las recomendaciones y, en definitiva, el papel desempeñado por la ADCA para contribuir a mejorar la regulación del sector cultural en Andalucía, y por tanto, a un mejor funcionamiento de los mercados en el sector cultural.

\section{Resultados}

\subsection{Resultados generales}

A continuación, se muestra el análisis de las tres funciones básicas de la ADCA: en primer lugar, determinar si las normas respetan los principios de la buena regulación; en segundo lugar, si inciden negativamente en la unidad de mercado; y en tercer lugar, si, con su aplicación, se erigen barreras que afectan a la competencia. Este examen se realiza durante las dos etapas que definen el rango de acción de la Agencia: una etapa inicial que comprende los años 2008-2013 (Ley 6/2007), y la etapa actual (2014-2019) donde la ADCA refuerza las tareas de asesoramiento a la Administración de la Junta de Andalucía.

En esta segunda etapa (2014-2018), se evidencia una mayor afluencia de informes relacionados con la LGUM, y en menor medida, los relativos a una mejora de la regulación, que contrastan con la etapa inicial, cuando la mayor parte de estos informes se referían a las grandes superficies (Gráfico 2).

\section{GRÁFICO 2}

\section{EVOLUCIÓN DEL NÚMERO DE INFORMES NORMATIVOS EMITIDOS} POR ADCA, 2008-2017

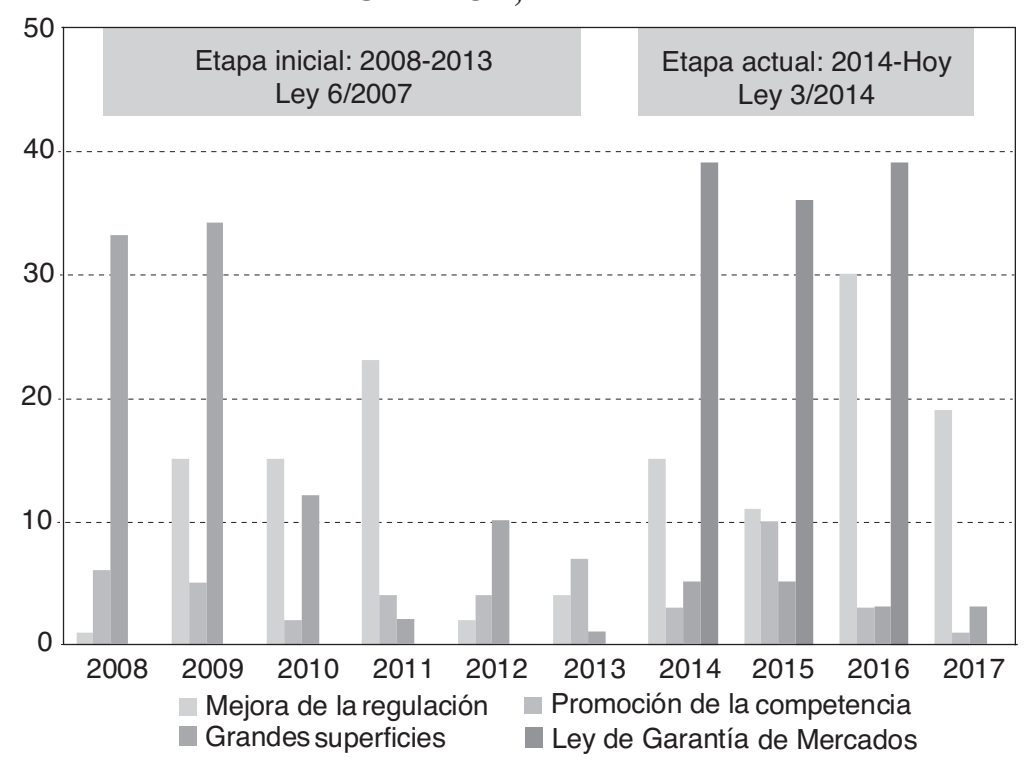

NOTA: Aunque el periodo de estudio es desde 2008 a 2018, solo se logró recoger información hasta 2017. FUENTE: Elaboración propia con base en ADCA. 
Pero, ¿cuál es la importancia de la cultura como ámbito de intervención de la ADCA? De los informes normativos presentados entre 2008 y 2019, 12 informes se relacionan directamente con el ámbito cultural, lo que representa el 7,8\% del total de la actividad de la Agencia (Gráfico 3), aunque se concentran entre 2016 y 2019. Se debe mencionar que esta cifra aumentaría si se consideraran los informes en ámbitos como la educación (como sería la regulación que involucra la educación cultural en el sistema educativo andaluz).

\section{GRÁFICO 3 \\ NÚMERO DE INFORMES NORMATIVOS EMITIDOS POR ADCA SEGÚN ÁMBITO, 2008-2019}

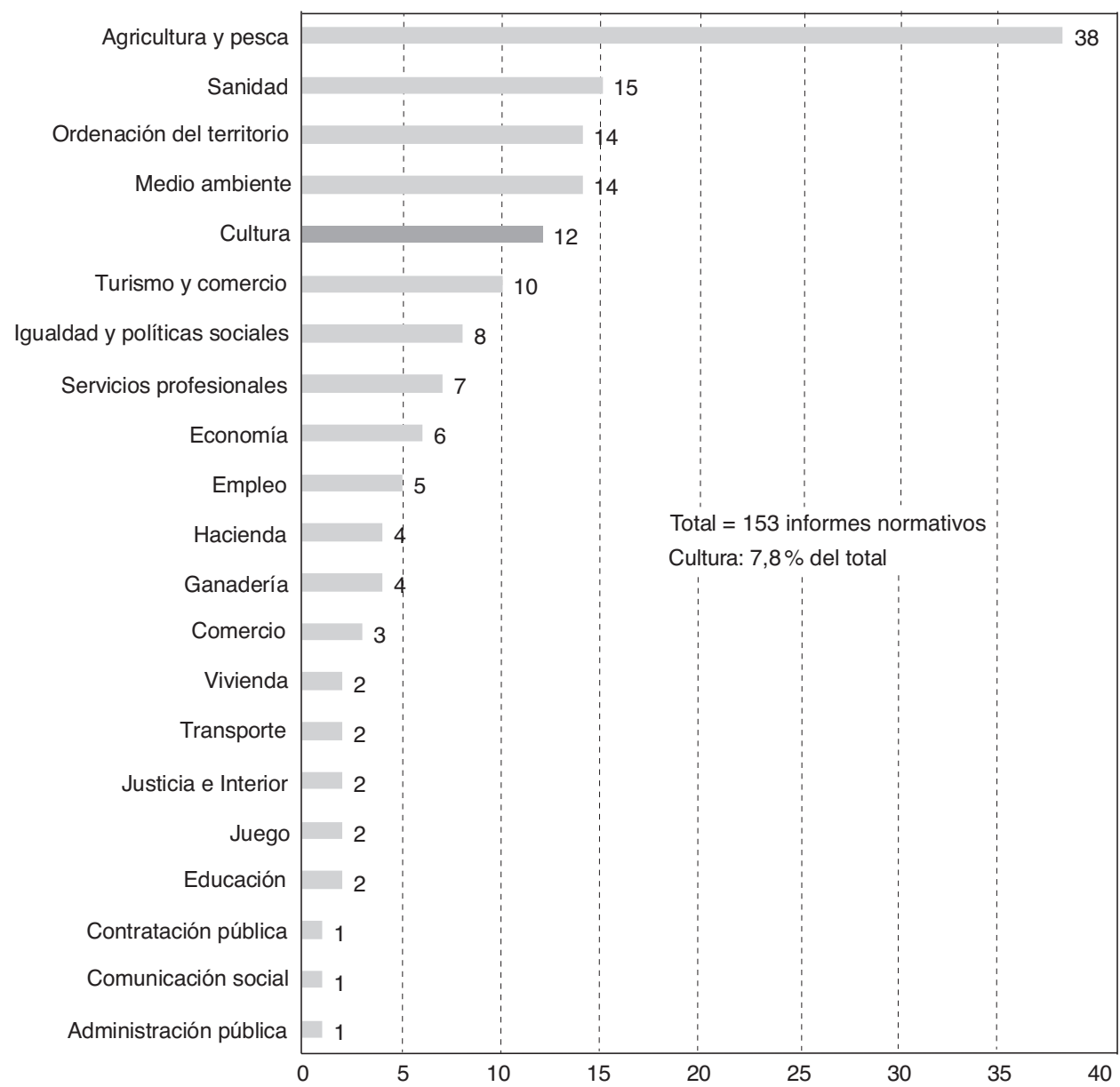

NOTA: Se han podido recopilar informes normativos hasta 2019.

FUENTE: Elaboración propia con base en ADCA. 
A continuación, se observa que el grueso de la actividad normativa en el ámbito cultural tiene lugar en 2007 y 2008, cuando se aprobaron seis leyes (que no contaron con informes de la ADCA), y luego se reactivó en el periodo 2016-2018 con cuatro leyes más. Mientras que en la primera etapa se reguló a favor de la radio y televisión de titularidad autonómica gestionada por la RTVA, el patrimonio histórico, la actividad museística y otras conexas como la educación cultural ${ }^{1}$, en la fase más reciente se aprobaron regulaciones para garantizar la prestación del servicio de televisión digital terrestre, la de fomento del emprendimiento en la región de Andalucía, y las que serán objeto de análisis en el siguiente apartado: Ley del Cine y Ley Audiovisual (Gráfico 4).

\section{GRÁFICO 4}

\section{LEYES EN EL ÁMBITO CULTURAL APROBADAS EN ANDALUCÍA, 1983-2018}

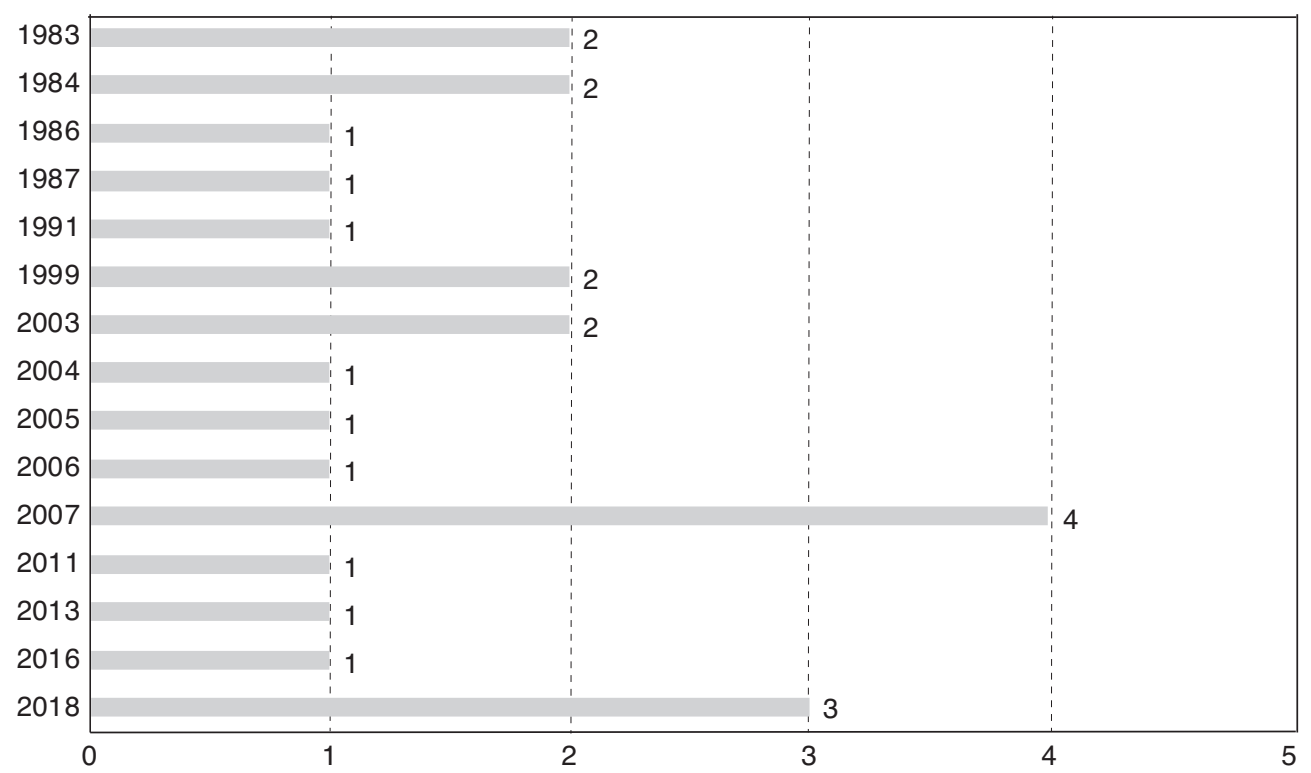

FUENTE: Elaboración propia con base en ADCA.

Habría que destacar, por consiguiente que, a pesar de la promulgación de normativa en materia cultural, el primer informe de recomendaciones de la ADCA a una propuesta por parte de las AAPP tiene lugar en 2016, fecha a partir de la cual la

${ }^{1}$ Ley 18/2007, de 17 de diciembre, de la radio y televisión de titularidad autonómica gestionada por

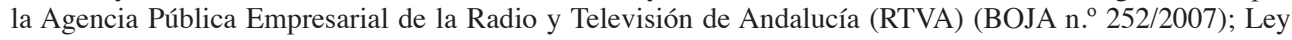
14/2007, del Patrimonio Histórico de Andalucía (BOJA n. 248/ 2007; BOE n. ${ }^{\circ}$ 38/2008); Ley 8/2007, de Museos y Colecciones Museográficas de Andalucía (BOJA n. . 205/2007); Ley 17/2007, de Ley de Educación de Andalucía (BOJA n. . 252/2007); Ley 5/2007, de creación del Instituto Andaluz del Patrimonio Histórico (BOJA n. ${ }^{\circ}$ 131/2007); Ley 55/2007 del Cine (Nacional) (BOE n. ${ }^{\circ}$ 312/2007). 
participación del sector cultural y patrimonio en el total de informes normativos ha venido aumentando, aún si se incluyen las cifras provisionales de 2019 (Gráfico 5).

Esto ha significado un cambio notorio en la regulación cultural si se compara con años previos; en este sentido, la ausencia de propuestas normativas informadas por la ADCA con antelación a 2016 puede interpretarse como el resultado de una gestión tradicional del sector cultural, asumido como un sector al margen del mercado.

\section{GRÁFICO 5}

\section{INFORMES NORMATIVOS EMITIDOS POR ADCA EN EL SECTOR DE CULTURA Y PATRIMONIO}

(En \%)

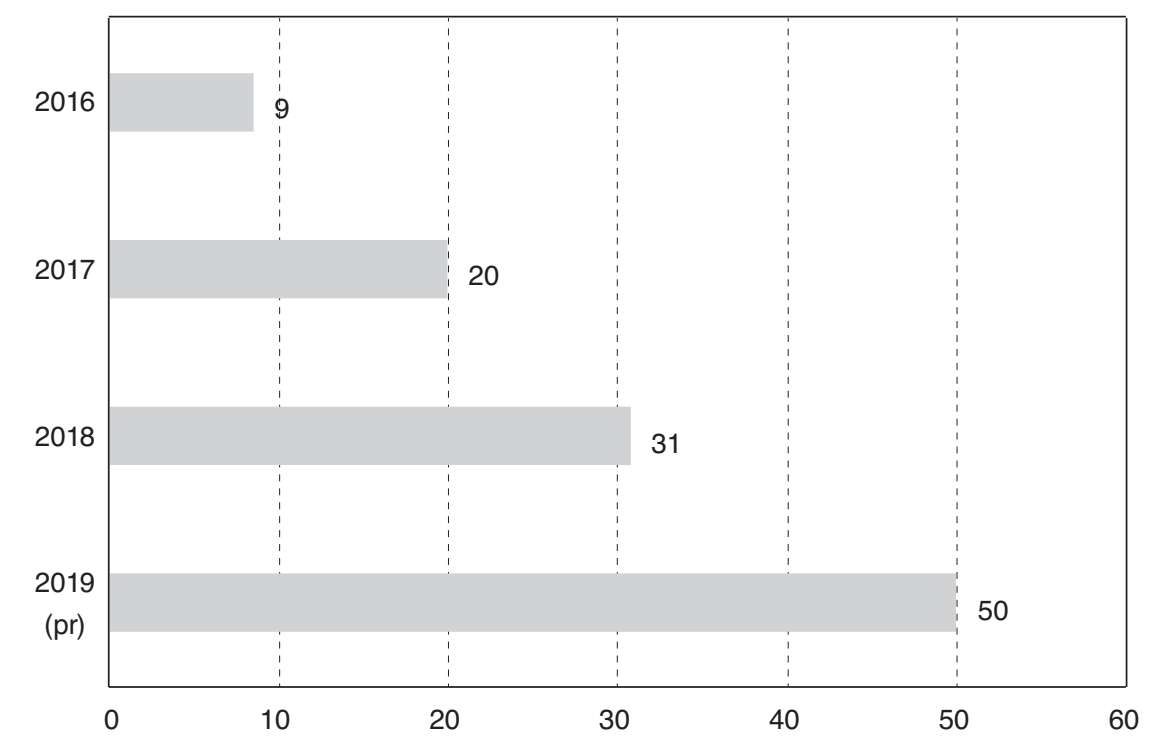

FUENTE: Elaboración propia con base en ADCA.

\subsection{Análisis de los proyectos normativos}

De los 12 proyectos objeto de informe por parte de la ADCA, se analizan 9, entre los cuales se encuentran los materializados en leyes y otras disposiciones posteriores, como son los casos de la Ley de Audiovisual y la Ley del Cine, o las resoluciones definitivas de órdenes aprobadas con posterioridad. Quedan al margen aquellos que han informado normas aún no promulgadas. Nos referimos a los informes N8/2018 sobre el anteproyecto de Ley de Artes Escénicas de Andalucía, el N11/2018 sobre el anteproyecto de Ley por el que se modifica la Ley 14/2007 de Patrimonio Histórico de Andalucía, y el proyecto de orden por el que se establecen las bases reguladoras de concesión de subvenciones, en régimen de concurrencia competitiva, a la producción de largometrajes, documentales y otras obras audiovisuales en Andalucía, informe N2/2019 (Cuadro 2). 
CUADRO 2

INFORMES NORMATIVOS EN SECTORES AUDIOVISUAL

Y ARTES ESCÉNICAS, 2016-2019

\begin{tabular}{|c|c|c|c|c|c|c|}
\hline Informe & Solicitud & Ámbito & Orientado a & $\begin{array}{c}\text { No se } \\
\text { cumple }\end{array}$ & $\begin{array}{c}\text { Sí se } \\
\text { cumple }\end{array}$ & $\begin{array}{c}\text { Parcial- } \\
\text { mente }\end{array}$ \\
\hline $\begin{array}{l}\text { Informe } \\
11 / 2016\end{array}$ & $\begin{array}{l}\text { Anteproyecto de } \\
\text { Ley Audiovisual } \\
\text { de Andalucía }\end{array}$ & Audiovisual & Regulación & 4 & 5 & 1 \\
\hline $\begin{array}{l}\text { Informe } \\
25 / 2016\end{array}$ & $\begin{array}{l}\text { Proyecto de or- } \\
\text { den }\end{array}$ & $\begin{array}{l}\text { Teatro, la mú- } \\
\text { sica, danza y el } \\
\text { circo en Anda- } \\
\text { lucía }\end{array}$ & $\begin{array}{l}\text { Bases regulado- } \\
\text { ras de concesión } \\
\text { de subvenciones }\end{array}$ & 1 & 3 & 0 \\
\hline $\begin{array}{l}\text { Informe } \\
28 / 2016\end{array}$ & $\begin{array}{l}\text { Proyecto de } \\
\text { orden }\end{array}$ & $\begin{array}{l}\text { Distribución y } \\
\text { promoción de } \\
\text { películas cine- } \\
\text { matográficas de } \\
\text { largometrajes en } \\
\text { salas de exhibi- } \\
\text { ción locales }\end{array}$ & $\begin{array}{l}\text { Bases regulado- } \\
\text { ras de concesión } \\
\text { de subvenciones }\end{array}$ & 0 & 4 & 1 \\
\hline $\begin{array}{l}\text { Informe } \\
2 / 2017\end{array}$ & $\begin{array}{l}\text { Proyecto de or- } \\
\text { den }\end{array}$ & Documentales & $\begin{array}{l}\text { Bases regulado- } \\
\text { ras sobre conce- } \\
\text { sión de subven- } \\
\text { ciones }\end{array}$ & 1 & 5 & 1 \\
\hline $\begin{array}{l}\text { Informe } \\
3 / 2017\end{array}$ & $\begin{array}{l}\text { Proyecto de or- } \\
\text { den }\end{array}$ & Cortometrajes & $\begin{array}{l}\text { Bases regulado- } \\
\text { ras de concesión } \\
\text { de subvenciones }\end{array}$ & 2 & 6 & 0 \\
\hline $\begin{array}{l}\text { Informe } \\
4 / 2017\end{array}$ & $\begin{array}{l}\text { Proyecto de or- } \\
\text { den }\end{array}$ & $\begin{array}{l}\text { Promoción del } \\
\text { tejido profesio- } \\
\text { nal del Flamen- } \\
\text { co }\end{array}$ & $\begin{array}{l}\text { Bases regulado- } \\
\text { ras de concesión } \\
\text { de subvenciones }\end{array}$ & 0 & 4 & 0 \\
\hline $\begin{array}{l}\text { Informe } \\
7 / 2017\end{array}$ & $\begin{array}{l}\text { Anteproyecto de } \\
\text { Ley del Cine de } \\
\text { Andalucía }\end{array}$ & Cine & Regulación & 2 & 13 & 3 \\
\hline $\begin{array}{l}\text { Informe } \\
5 / 2018\end{array}$ & $\begin{array}{l}\text { Proyecto de de- } \\
\text { creto }\end{array}$ & $\begin{array}{l}\text { Reglamento ge- } \\
\text { neral admisión } \\
\text { de personas en } \\
\text { los estableci- } \\
\text { mientos de es- } \\
\text { pectáculos pú- } \\
\text { blicos y activi- } \\
\text { dades recreati- } \\
\text { vas }\end{array}$ & Regulación & 1 & 6 & 0 \\
\hline $\begin{array}{l}\text { Informe } \\
10 / 2018\end{array}$ & $\begin{array}{l}\text { Proyecto de or- } \\
\text { den }\end{array}$ & $\begin{array}{l}\text { Microempresas, } \\
\text { pequeñas y me- } \\
\text { dianas empre- } \\
\text { sas culturales y } \\
\text { creativas }\end{array}$ & $\begin{array}{l}\text { Bases regulado- } \\
\text { ras de concesión } \\
\text { de subvenciones }\end{array}$ & 3 & 2 & 1 \\
\hline
\end{tabular}

NOTA: Los valores de las columnas corresponden a las recomendaciones incluidas en los informes y su seguimiento se analiza en los gráficos 6 y 7.

FUENTE: Elaboración propia con base en ADCA. 
Los principales resultados encontrados son los siguientes:

1. En primer lugar, como hemos expuesto antes, los informes en materia de cultura se realizan en el periodo 2016-2019, lo que indica que la ampliación de funciones de la ADCA se tradujo en una mayor demanda de sus actuaciones expresada mediante los informes.

2. Un segundo resultado apunta a destacar tres sectores que concentran las actuaciones reguladoras de la ADCA:

- El sector audiovisual y de cine, con el $45 \%$ de la normativa existente, que comprende cinco informes, dos leyes aprobadas y tres resoluciones sobre bases reguladoras para la concesión de subvenciones en el sector del cine.

- El sector de artes escénicas, con tres informes que representan el $27 \%$ de la normativa total en el sector, repartidos en un anteproyecto de ley de artes escénicas y dos resoluciones sobre bases reguladoras de concesión de subvenciones al teatro, danza, y circo y a la promoción del tejido profesional de flamenco.

- Un grupo mixto conformado por las pymes culturales, patrimonio histórico y espectáculos públicos, cada una con el $9 \%$ de los informes emitidos. En relación con las pymes, se cuenta un informe sobre bases reguladoras para la concesión de subvenciones y apoyo a microempresas, y pequeña y mediana empresa creativa; en el segundo caso, un anteproyecto de ley de patrimonio histórico, y en el tercero, un informe sobre el proyecto de decreto por el que se modifica el reglamento general de admisión de personas en los establecimientos de espectáculos públicos y actividades recreativas.

3. El tercer resultado se refiere al seguimiento de las recomendaciones por parte de los centros directivos y su reflejo en la normativa aprobada y publicada en el BOJA por la Junta de Andalucía. Al analizar los tres sectores mencionados, se observa el alto compromiso con el seguimiento de las recomendaciones emanadas. En particular, se destaca la mayor incorporación de recomendaciones en las resoluciones para la concesión de subvenciones, 71\% (el 79\% si se incluye la recogida parcial de recomendaciones), seguidas por la normativa con rango de ley con seguimiento del $64 \%$ (el 79\%, si se considera el seguimiento parcial). Esto significa que, en los casos de artes escénicas y cine, ha habido un alto seguimiento a las recomendaciones de la Agencia, muy superior al que se registra en el resto de sectores y ámbitos de acción de la ADCA, cuyo seguimiento es del $31 \%$ (Gráfico 6). 


\section{GRÁFICO 6}

\section{CUMPLIMIENTO DE RECOMENDACIONES DE INFORMES NORMATIVOS EN SECTORES AUDIOVISUAL Y ARTES ESCÉNICAS, 2016-2019}

(En \%)

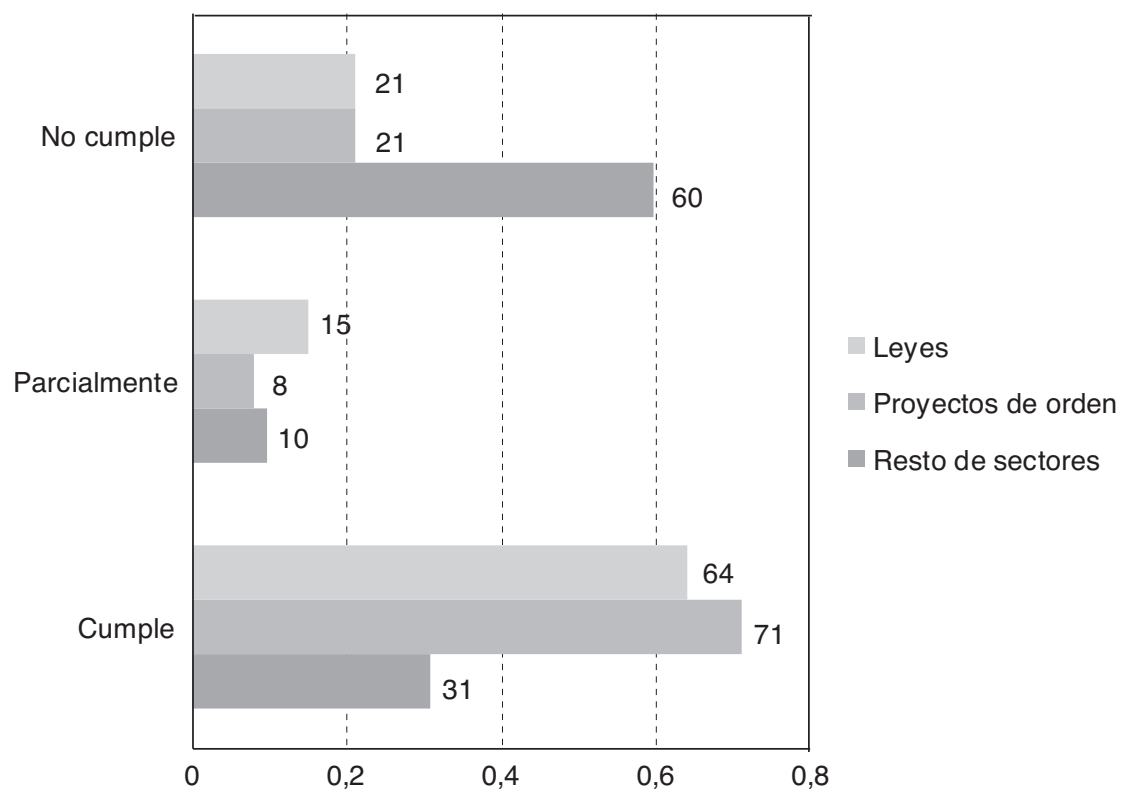

FUENTE: Elaboración propia con base en informes normativos de ADCA.

En el caso de las regulaciones en el sector audiovisual y del cine, se observa una alta proporción de recomendaciones recogidas en la actual Ley del Cine, $72 \%$ (si se amplía a la adopción parcial de las mismas, reflejaría un seguimiento del 89\%), muy por encima de las adoptadas en la Ley de Audiovisual, cuyo grado de seguimiento es solo del $50 \%$. El incumplimiento de las recomendaciones en esta última ley es notorio, aunque inferior si se compara con el resto de sectores de intervención de la ADCA (60\%) (Gráfico 7).

4. En cuanto a los problemas de competencia que más afectan al sector cultural, podemos señalar la limitación al ejercicio de la actividad económica y restricciones de acceso a los participantes en el mercado por razón de la naturaleza del operador económico y de orden geográfico (Gráfico 8). El subsector del cine ofrece un caso representativo de este tipo de barreras. Entre las más frecuentes detectadas por la ADCA en sus informes normativos destacan la restricción a la competencia por razones de territorialidad, la cual opera por dos vías: por una primera, al inclinar los criterios de valoración en la concesión de subvenciones hacia aquellas empresas con sede o establecimiento en Andalucía (Informe N2/2017, N3/2017, N28/2016), y por una segunda vía, 


\section{GRÁFICO 7}

\section{CUMPLIMIENTO DE RECOMENDACIONES DE INFORMES NORMATIVOS SOBRE ANTEPROYECTOS DE LEY EN AUDIOVISUALES Y ARTES ESCÉNICAS, 2016-2019}

(En \%)

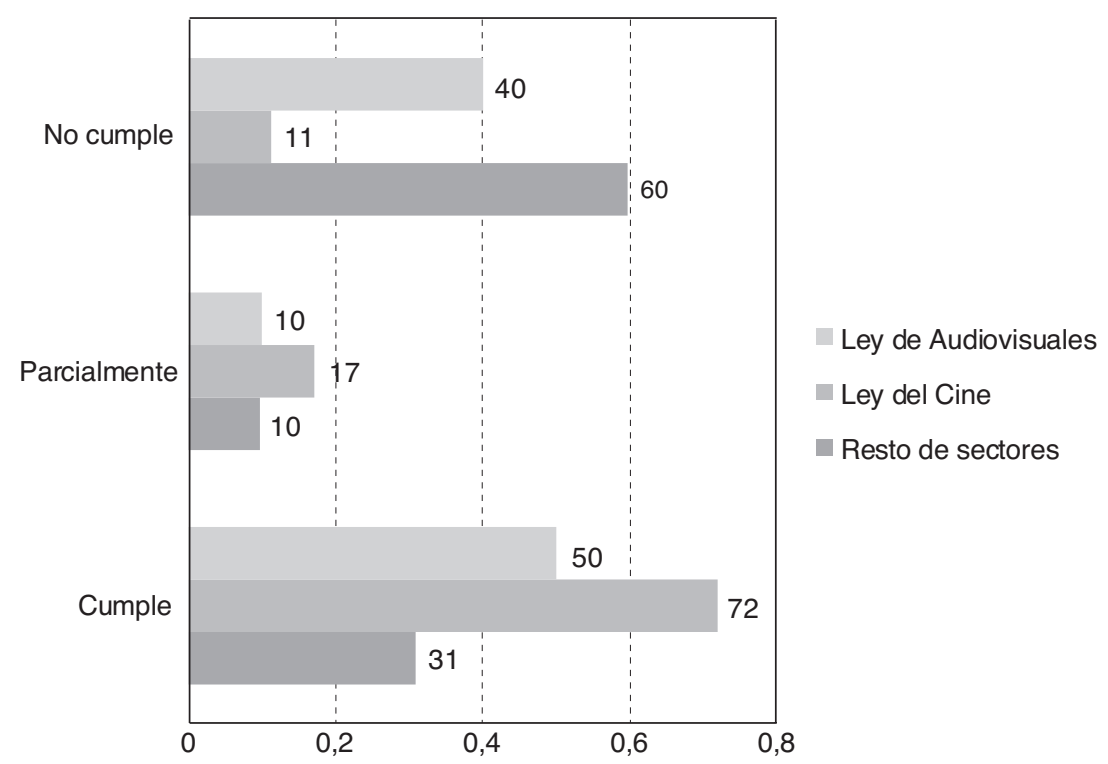

FUENTE: Elaboración propia con base en informes normativos de ADCA.

restringiendo el acceso al mercado por parte de determinados operadores en razón a su naturaleza de persona física o jurídica, lo que puede revestir un carácter discriminatorio en el caso de requisitos para los solicitantes (Informe No2/2017, N28/2016).

En ambos casos, estos problemas han sido subsanados en la normativa publicada con posterioridad por el centro proponente, como son los casos de la Orden de 19 de mayo de 2017, por la que se establecen las bases reguladoras de concesión de subvenciones en régimen de concurrencia competitiva a la producción de documentales en Andalucía (BOJA n. ${ }^{\circ} 98$ de 25 de mayo de 2017), la Orden de 19 de mayo de 2017, por la que se establecen las bases reguladoras de concesión de subvenciones en régimen de concurrencia competitiva, a la producción de cortometrajes en Andalucía (BOJA n. ${ }^{\circ}$ 99, 26 de mayo de 2017) y la Orden de 7 de febrero de 2017, por la que se establecen las bases reguladoras de concesión de subvenciones, en régimen de concurrencia competitiva para favorecer la distribución y promoción de películas cinematográficas de largometraje en salas de exhibición cinematográfica de Andalucía (BOJA n. ${ }^{\circ}$ 37, de 23 de febrero de 2017). 


\section{GRÁFICO 8 \\ PROBLEMAS DE COMPETENCIA DETECTADOS EN LOS INFORMES NORMATIVOS EN EL SECTOR CULTURA Y PATRIMONIO, Y RESTO DE SECTORES}

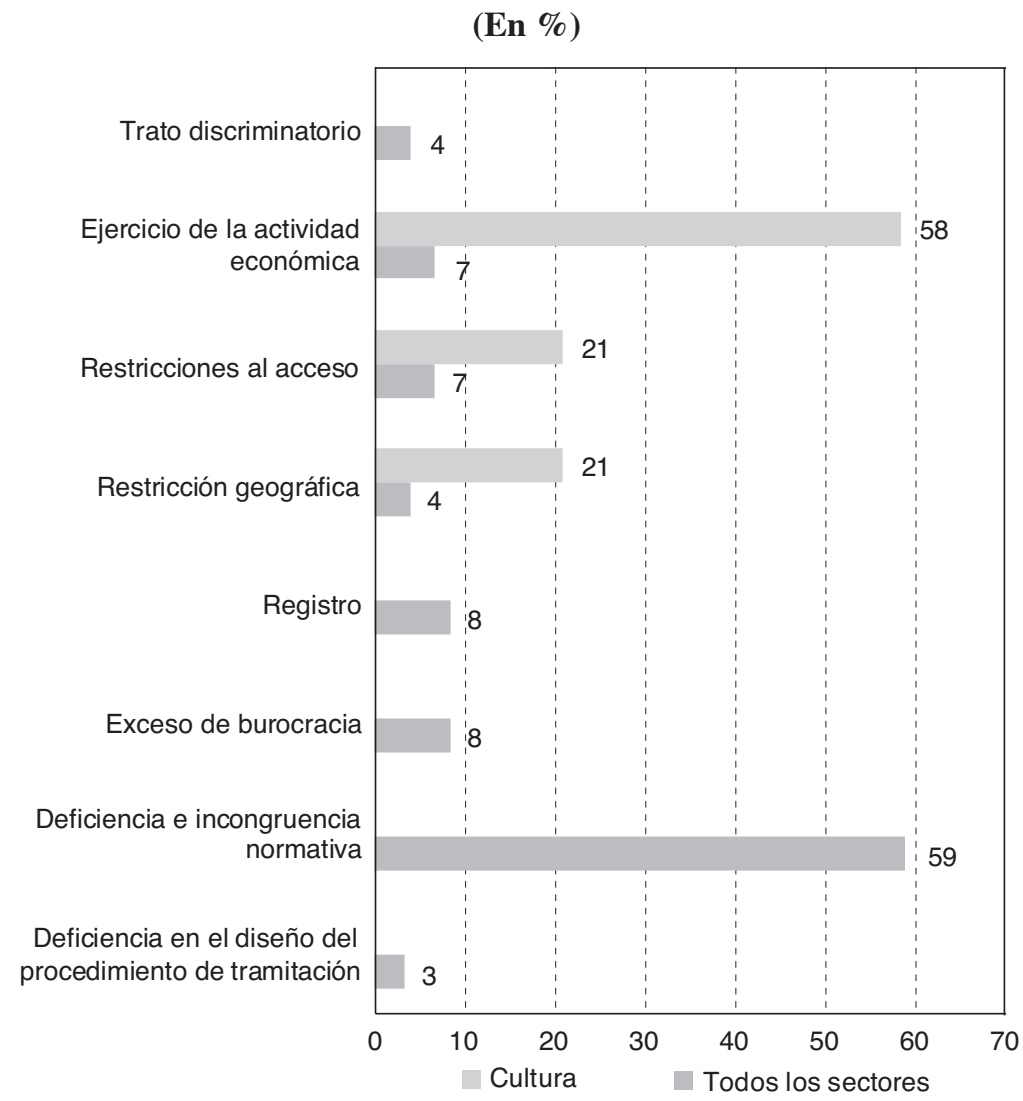

FUENTE: Elaboración propia con base en informes normativos de ADCA.

Se debe precisar que, a pesar de los esfuerzos por mejorar la promoción de la competencia en el sector cultural, en algunos casos las recomendaciones no han sido atendidas, como sucede con los proyectos de orden y leyes referidas al sector audiovisual y al de artes escénicas (Gráficos 6 y 7). Al respecto, un caso frecuente suele ser el de los criterios relativos a la experiencia profesional previa de los solicitantes, aplicados para la concesión de subvenciones, que son señalados como problemas de competencia que limitan el ejercicio a la actividad económica (Gráfico 8).

Aunque la ADCA ha sugerido -y suele recomendar- la eliminación de estos criterios, que otorgan ventajas a las empresas establecidas sobre los nuevos operadores, no han sido acogidos en ningún caso por los centros directivos. En respuesta a los informes normativos de la Agencia, la tendencia ha sido reducir 
el peso asignado a esta experiencia en las convocatorias y consecuentemente elevar la importancia de criterios artísticos, de calidad u originalidad del proyecto. En todo caso, este tipo de seguimiento evidencia el papel clave de la Agencia en la promoción de la competencia en el sector cultural y en el sector audiovisual, en particular, donde un instrumento de política cultural como es la regulación está favoreciendo el mejor funcionamiento del mercado del cine.

En el análisis realizado destaca que las propuestas de regulación cultural no registran problemas, tales como el exceso de burocracia de la administración propuesta. Adicionalmente, estas propuestas normativas en cultura no muestran deficiencia alguna en el planteamiento de la regulación ni en el diseño del procedimiento de tramitación, la cual se ha agilizado, favoreciendo el acceso de operadores al proporcionar un procedimiento de otorgamiento abierto, con posibilidad de obtención de formularios por medios electrónicos, así como su presentación y conocimiento de la tramitación por esta vía (Informe n. ${ }^{\circ}$ 4/2017). Sin embargo, cuando se analiza la frecuencia de problemas de competencia en general, hallados en los informes normativos, el sector cultural supera el promedio del resto de ámbitos (Gráfico 9).

GRÁFICO 9

\section{NÚMERO DE PROBLEMAS DE COMPETENCIA DETECTADOS EN CADA INFORME NORMATIVO EMITIDO POR LA ADCA, 2016-2018}

(En \%)

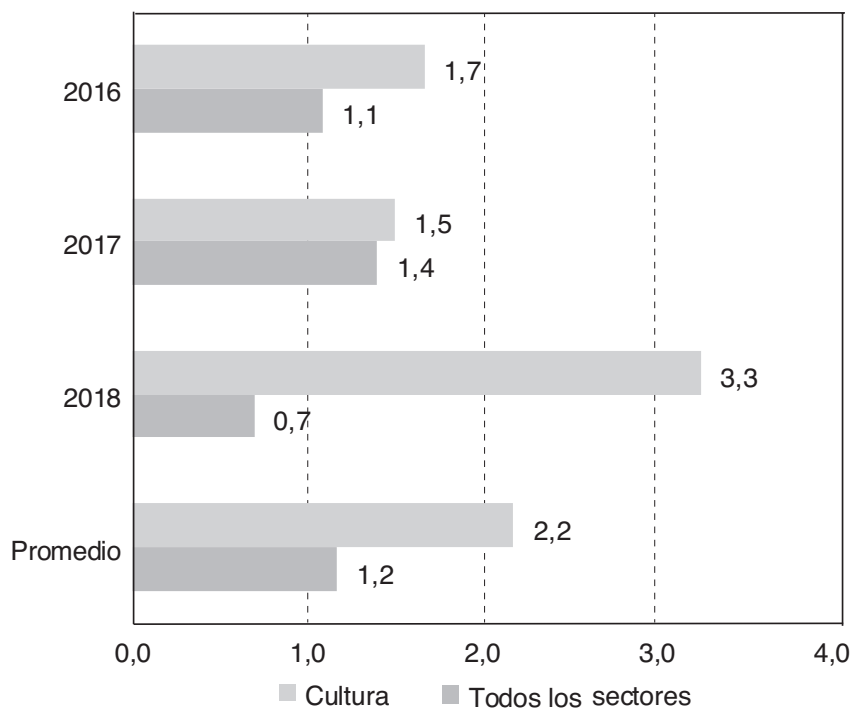

NOTA: El indicador anual se obtiene dividiendo el número de problemas encontrados en cada año entre el número de informes anual. Entre los problemas de competencia contados se hallan deficiencia, incongruencia normativa, exceso de burocracia, registro, restricción geográfica, restricciones al acceso, ejercicio de la actividad económica, y trato discriminatorio.

FUENTE: Elaboración propia con base en la Agencia de Defensa de la Competencia de Andalucía, ADCA. 
5. En relación a los principios de buena regulación afectados, sobresale el de seguridad jurídica, que ha dado lugar al $37 \%$ de las recomendaciones realizadas a la Administración Pública; le siguen los principios de transparencia, proporcionalidad y necesidad $(18 \%, 17 \%$ y $16 \%$ de recomendaciones, respectivamente). Los principios con menos recomendaciones son los de eficiencia, eficacia y accesibilidad, con menos del 7\% (Gráfico 10).

\section{GRÁFICO 10 \\ PRINCIPIOS DE BUENA REGULACIÓN AFECTADOS POR LA NORMATIVA \\ PROPUESTA, 2016-2018 \\ (En \%)}

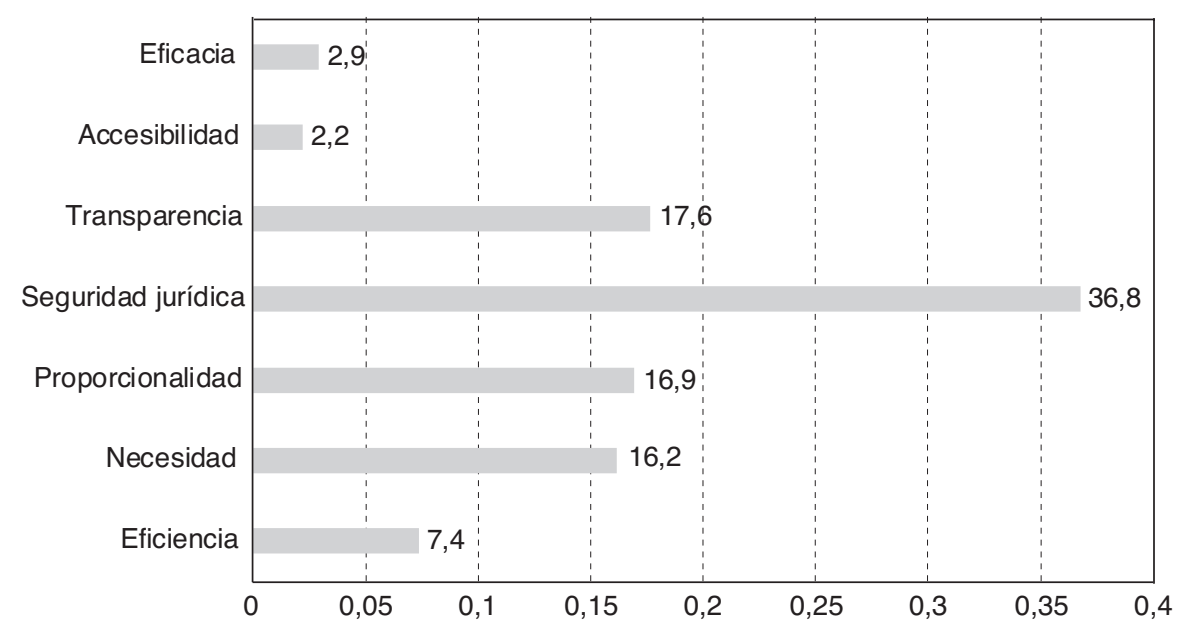

FUENTE: Elaboración propia con base en informes normativos de ADCA.

En los informes normativos sobre proyectos de órdenes en artes escénicas y audiovisuales se debe precisar que en el caso de las bases reguladoras para promover el tejido del flamenco, la incorporación a la norma ha sido del $100 \%$, seguido de un seguimiento del $75 \%$ en el caso de las bases reguladoras para la promoción del teatro, música, danza y circo, y por encima del $70 \%$ en las resoluciones para la concesión de subvenciones a documentales (71\%), cortometrajes (75\%) y largometrajes $(80 \%)$. En este último caso, si se suma la adopción parcial en la normativa, el grado de cumplimiento llegaría al $100 \%$. Por último, se debe subrayar la baja incorporación de recomendaciones a la normativa en las subvenciones a las microempresas, y pequeñas y medianas empresas culturales y creativas, con solo 33\% (Gráfico 11). 


\section{GRÁFICO 11 \\ CUMPLIMIENTO DE RECOMENDACIONES DE INFORMES NORMATIVOS SOBRE PROYECTOS DE ORDEN EN AUDIOVISUALES Y ARTES ESCÉNICAS, 2016-2019}

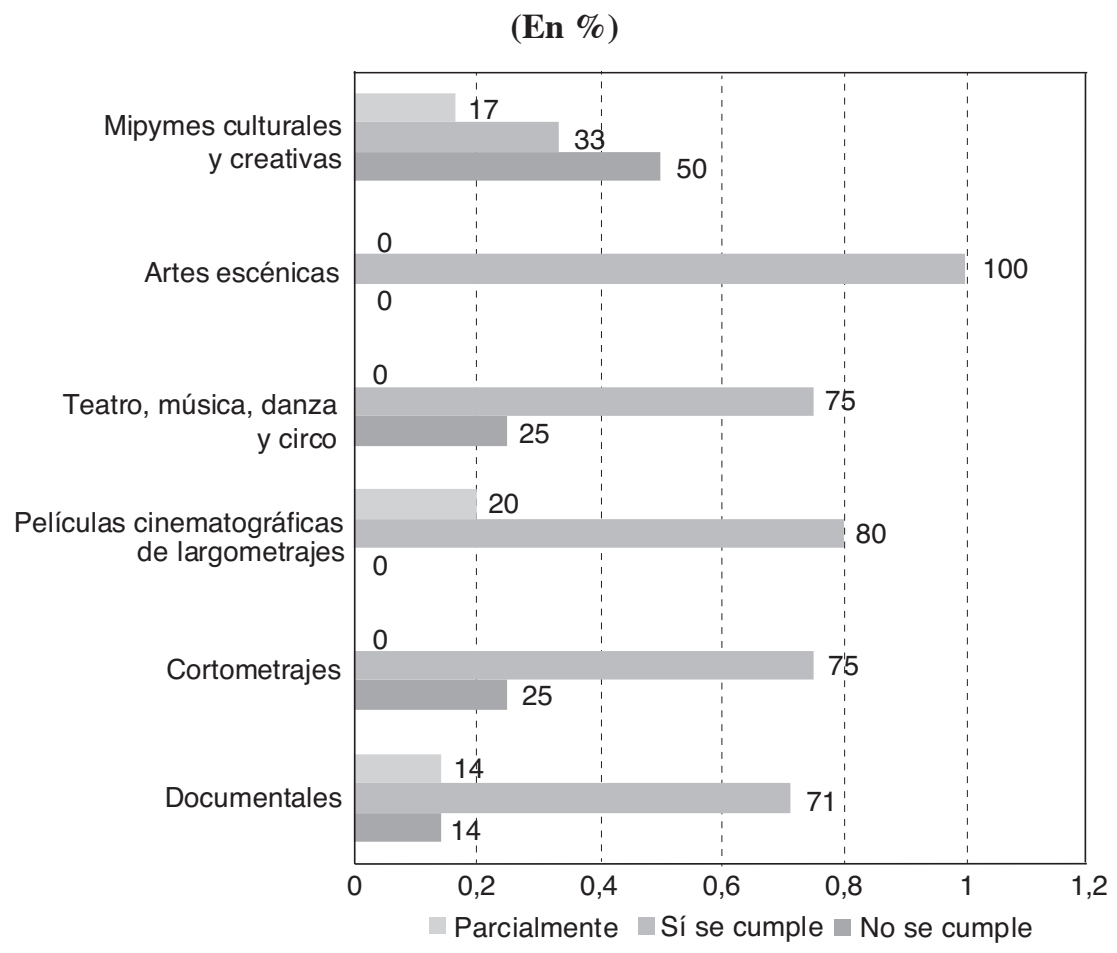

FUENTE: Elaboración propia con base en ADCA. Informes Normativos N 2/2017, N 3/2017, N 28/2016, N25/2016, N 4/2017, N 10/2018.

\section{Conclusiones}

La calidad de la regulación es un elemento clave para favorecer un funcionamiento eficiente de las actividades económicas. La regulación económica es una herramienta que puede contribuir a incrementar el bienestar social cuando resuelve los fallos del mercado y promueve la competencia en los mercados.

Uno de los instrumentos más destacados para mejorar la calidad de la regulación lo constituye el control ex ante de los proyectos normativos, máxime si se tiene en cuenta que, en ocasiones, la propia actuación de los poderes públicos introduce restricciones o trabas innecesarias y desproporcionadas al acceso o ejercicio de las actividades económicas, lo cual afecta o distorsiona el libre juego competitivo de los operadores económicos. 
En Andalucía, a este respecto, un factor clave ha sido concentrar las competencias y actuaciones para la mejora de la regulación económica en un organismo especializado, la ADCA, que ejerce esta función desde la aprobación de la Ley 3/2014. Esto ha significado en la práctica apostar por una agencia independiente, que ya contaba con las funciones de promoción y defensa de la competencia de los mercados. En este sentido, el año 2014 representa un punto de inflexión tanto en la ampliación de funciones de la Agencia como en su incidencia efectiva en el sector cultural, en relación a la aplicación de los principios de Better and Smart Regulation.

Por lo que se refiere al ámbito específico de la regulación cultural, se constata que el primer proyecto normativo informado por la ADCA data de 2016 y se concentra en tres sectores: audiovisual, patrimonio y artes escénicas. Esto denota la escasa preocupación, con anterioridad a esa fecha, por fomentar la competencia en el sector cultural, y refleja la noción de cultura como un conjunto de actividades al margen del mercado y con una escasa preocupación por alcanzar mayor eficiencia en la asignación de recursos.

En lo que respecta al seguimiento de las recomendaciones efectuadas a las leyes reguladoras en materia de cultura, cabe destacar especialmente la incorporación de un $72 \%$ de recomendaciones en la Ley 6/2018 de 9 de julio del Cine de Andalucía (BOJA n. ${ }^{\circ} 135$ de 13 de julio de 2018), así como del $50 \%$ en la Ley 10/2018 de 9 de octubre de audiovisual de Andalucía (BOE n. ${ }^{\circ} 269$ de 7 de noviembre de 2018), un seguimiento muy superior al de otros sectores económicos, que ronda el $40 \%$. Así mismo, se destaca el alto compromiso con el seguimiento de las recomendaciones emanadas de los informes normativos de la ADCA, en audiovisual y artes escénicas, alcanzando el $100 \%$ en este último sector.

En cuanto a los problemas que más afectan a la competencia en el sector cultural, se destaca la limitación al ejercicio de la actividad económica y restricciones de acceso a los mercados. Estos se focalizan en barreras territoriales y por naturaleza de los operadores potenciales, aspectos que pueden inducir a un trato discriminatorio de unos operadores frente a otros, pero que han sido subsanados en la normativa publicada con posterioridad por el centro proponente. La subsanación de principios que afectan a la competencia redundará a medio plazo en un mejor funcionamiento de estos mercados, cuya incidencia en su estructura habrá de ser objeto de atención con posterioridad.

El trabajo contribuye a la literatura sobre regulación y competencia en el sector cultural de Andalucía en dos aspectos. En primer lugar, se identificaron las principales restricciones que los proyectos normativos que regulan el sector cultural suelen incorporar desde la perspectiva de la competencia efectiva y la regulación eficiente. Estas restricciones, aunque identificadas para el sector cultural en Andalucía, son extrapolables a otros territorios y ámbitos de análisis.

En segundo lugar, se pueden observar buenas prácticas, aceptadas por los centros directivos, a partir de las recomendaciones realizadas por el CDC, en el escenario de un diálogo entre instituciones públicas con objetivos diversos pero que, en última instancia, tratan con sus iniciativas el logro de objetivos de interés general. En 
el caso que nos ocupa podrían circunscribirse, entre otros, a la mejor asignación de los recursos públicos y a la posibilidad de que una buena regulación contribuya a dinamizar un sector clave para la economía de Andalucía. Por último, cabe resaltar la necesidad de seguimiento de los anteproyectos de artes escénicas y patrimonio, aun no publicados, para seguir comprobando la eficacia del papel de la ADCA en la promoción de los principios de la buena regulación y competencia.

\section{Referencias bibliográficas}

Alcalá, F., \& Jiménez, F. (2018). Los costes económicos del déficit de calidad institucional y la corrupción en España. Informes 2018. Economía y Sociedad. Fundación BBVA. Madrid.

Buccirossi, P., Ciari, L., Duso, T., Spagnolo, S., \& Vitale, C. (2013). Competition Policy and Productivity Growth: An Empirical Assessment. The Review of Economics and Statistics 95(4),1324-36. Recuperado de http://www.mitpressjournals.org/doi/abs/10.1162/REST_a_00304\#.U75kjvmSyVM.

Clougherty, J. (2010). Competition Policy Trends and Economic Growth: Cross-National Empirical Evidence. International Journal of the Economics of Business 17(1), 111-27.

Consejo de Defensa de la Competencia de Andalucía (2016). Informe N. ${ }^{\circ} 11 / 2016$, sobre el anteproyecto de ley audiovisual de Andalucía.

Consejo de Defensa de la Competencia de Andalucía (2016). Informe N. ${ }^{\circ}$ 25/2016, sobre el proyecto de orden por la que se establecen las bases reguladoras de concesión de subvenciones en régimen de concurrencia competitiva para la promoción del teatro, la música, la danza y el circo en Andalucía.

Consejo de Defensa de la Competencia de Andalucía (2016). Informe N. . 28/2016, sobre el proyecto de orden por la que se establecen las bases reguladoras de concesión de subvenciones en régimen de concurrencia competitiva para favorecer la distribución y promoción de películas cinematográficas de largometraje en salas de exhibición cinematográfica de Andalucía.

Consejo de Defensa de la Competencia de Andalucía (2017). Informe N. ${ }^{o}$ 2/2017, sobre el proyecto de orden por la que se establecen las bases reguladoras de la concesión de subvenciones en régimen de concurrencia competitiva a la producción de documentales en Andalucía.

Consejo de Defensa de la Competencia de Andalucía (2017). Informe N. ${ }^{\circ} 3 / 2017$, sobre proyecto de orden por la que se establecen las bases reguladoras para la concesión de subvenciones, en régimen de concurrencia competitiva a la producción de cortometrajes en Andalucía.

Consejo de Defensa de la Competencia de Andalucía (2017). Informe N. ${ }^{\circ} 4 / 2017$, sobre el proyecto de orden por la que se establecen las bases reguladoras de concesión de subvenciones, en régimen de concurrencia competitiva, para la promoción del tejido profesional del flamenco en Andalucía.

Consejo de Defensa de la Competencia de Andalucía (2017). Informe N. $7 / 2017$ sobre el anteproyecto de Ley del Cine de Andalucía.

Consejo de Defensa de la Competencia de Andalucía (2018). Informe N. ${ }^{o}$ 5/2018, sobre el proyecto de decreto por el que se modifica el reglamento general de la admisión de personas en los establecimientos de espectáculos públicos y actividades recreativas, aprobado por el decreto 10/2003, de 28 de enero. 
Consejo de Defensa de la Competencia de Andalucía (2018). Informe N. ${ }^{o} 8 / 2018$, sobre el anteproyecto de Ley de Artes Escénicas de Andalucía.

Consejo de Defensa de la Competencia de Andalucía (2018). Informe N. ${ }^{\circ}$ 10/2018, sobre el proyecto de orden por la que se aprueban las bases reguladoras para la concesión de subvenciones, en régimen de concurrencia competitiva, de apoyo a las microempresas, pequeñas y medianas empresas culturales y creativas andaluzas, para el fomento de su competitividad, modernización e internacionalización.

Consejo de Defensa de la Competencia de Andalucía (2018). Informe N. 11/2018, sobre el anteproyecto de ley por el que se modifica la ley 14/2007, de 26 de noviembre, de patrimonio histórico de Andalucía.

Consejo de Defensa de la Competencia de Andalucía (2019). Informe N. ${ }^{o} 2 / 2019$, sobre el proyecto de orden por la que se establecen las bases reguladoras de concesión de subvenciones, en régimen de concurrencia competitiva, a la producción de largometrajes, documentales y otras obras audiovisuales en Andalucía.

Decreto-ley 5/2014, de 22 de abril, de medidas normativas para reducir las trabas administrativas para las empresas. Boletín Oficial de la Junta de Andalucía, 30 de abril de 2014, num.82, pp. 7-51.

Extracto de la Resolución de 30 de mayo de 2018, de la Dirección de la Agencia Andaluza de Instituciones Culturales, por la que se convocan para el año 2018 las subvenciones para la promoción del teatro, la música, la danza y el circo en Andalucía. Boletín Oficial de la Junta de Andalucía, 7 de junio de 2018, num.109, pp.225-227.

Extracto de la Resolución de 30 de mayo de 2018, de la Dirección de la Agencia Andaluza de Instituciones Culturales, por la que se convocan para el año 2018 las subvenciones para la promoción del tejido profesional del flamenco en Andalucía. Boletín Oficial de la Junta de Andalucía, 7 de junio de 2018, num. 109, pp. 228-229.

Extracto de la Resolución de 30 de mayo de 2018, de la Dirección de la Agencia Andaluza de Instituciones Culturales, por la que se convocan para el año 2018 las ayudas previstas en la Orden de 1 de agosto de 2016, por la que se establecen las bases reguladoras de concesión de subvenciones, en régimen de concurrencia competitiva, a la producción de largometrajes en Andalucía. Boletín Oficial de la Junta de Andalucía, 8 de junio de 2018, núm 110, pp. 35-36.

Fiori, G., Nicoletti, G., Scarpetta, S., \& Schiantarelli, F. (2012). Employment Effects of Product and Labour Market Reforms: Are There Synergies? The Economic Journal, 122(558), F79-F104. Recuperado de http://onlinelibrary.wiley.com/doi/10.1111/j.14680297.2011.02494.x/full

Gutmann, J., \& Voigt, S. (2014). Lending a Hand to the Invisible Hand? Assessing the Effects of Newly Enacted Competition Laws. Recuperado de https://papers.ssrn.com/sol3/ papers.cfm?abstract_id $=2392780$

Guía para la mejora de la regulación económica en Andalucía (2017). Recuperado de http:// web.adca.junta-andalucia.es/mejora-de-la-regulacion/guia-para-la-mejora-de-la-regulacion-economica-y-formularios.

Kronthaler, F. (2010). Factors Influencing the Implementation of Recently Enacted Competition Laws: An Empirical Analysis. International Research Journal of Finance and Economics 51,71-87.

Ley 20/2013, de 9 de diciembre, de garantía de la unidad de mercado. Boletín Oficial del Estado, 10 de diciembre de 2013, núm. 295, pp. 97953-97978. 
Ley 6/2018, de 9 de julio, del Cine de Andalucía. Boletín Oficial del Estado, 2 de agosto de 2018, núm. 186,pp. 77671-77702.

Ley de Cine de Andalucía. Boletín Oficial de la Junta de Andalucía, 13 de julio de 2018, núm. 135, pp. 9-40.

Ley 10/2018, de 9 de octubre, audiovisual de Andalucía, Boletín Oficial del Estado, 7 de noviembre de 2018, núm. 269, pp. 108238-108290.

Mandelkern Group on Better Regulation (2001). Final Report, recuperado de http://ec.europa.eu/smart-regulation/better_regulation/documents/mandelkern_report.pdf.

Ministerio de Cultura y Deporte (2018). Anuario de Estadísticas Culturales. División de Estadística y Estudios, Secretaría General Técnica, Madrid.

Nickell, S. (1996). Competition and Corporate Performance. Journal of Political Economy 104(4), 724-746.

OCDE (2014). Factsheet on how competition policy affects macro-economic outcomes. Recuperado de: www.oecd.org/daf/competition

Orden de 24 de marzo de 2017 por la que se establecen las bases reguladoras de concesión de subvenciones en régimen de concurrencia competitiva para la promoción del tejido profesional del flamenco en Andalucía. Boletín Oficial de la Junta de Andalucía, 29 de marzo de 2017, num. 60, pp. 13-43.

Orden de 21 de marzo de 2018 , por la que se establecen las bases reguladoras de concesión de subvenciones, en régimen de concurrencia competitiva, para la promoción del tejido asociativo del flamenco en Andalucía. Boletín Oficial de la Junta de Andalucía, 28 de marzo de 2018, num. 61, pp. 14-30.

Orden de 1 de agosto de 2016, por la que se establecen las bases reguladoras de concesión de subvenciones, en régimen de concurrencia competitiva, a la producción de largometrajes en Andalucía. Boletín Oficial de la Junta de Andalucía, 8 de agosto 2016, num.151, pp. 155-287.

Orden de 15 de octubre de 2018, por la que se aprueban las bases reguladoras para la concesión de subvenciones, en régimen de concurrencia competitiva, de apoyo a las microempresas, pequeñas y medianas empresas culturales y creativas, para el fomento de su competitividad, modernización e internacionalización. Boletín Oficial de la Junta de Andalucía, 19 de octubre de 2018, núm. 203,pp. 57-85.

Petersen, N. (2013). Antitrust Law and the Promotion of Democracy and Economic Growth. Journal of Competition Law \& Economics 9(3), 593-636.

Resolución de 1 de marzo de 2018, de la Dirección General de Bienes Culturales y Museos, por la que se somete al trámite de información pública el anteproyecto de ley por el que se modifica la Ley 14/2007, de 26 de noviembre, del Patrimonio Histórico de Andalucía. Boletín Oficial de la Junta de Andalucía, 7 de marzo de 2018, num. 46, pp. 211-212.

Resolución de 21 de marzo de 2018, de la Dirección General de Innovación Cultural y del Libro, por la que se acuerda la apertura del trámite de información pública del Anteproyecto de la Ley de las Artes Escénicas de Andalucía. Boletín Oficial de la Junta de Andalucía, 28 de marzo de 2018, num. 61, pp.14-30.

Resolución de 18 de julio de 2018, de la Dirección de la Agencia Andaluza de Instituciones Culturales, por la que se convocan para el año 2018 las subvenciones para la promoción de festivales flamencos de pequeño y mediano formato. Boletín Oficial de la Junta de Andalucía, 1 de agosto de 2018, num.148, pp. 30-39; pp. 40-41. 
Resolución de 19 de abril de 2016, de la Agencia de Defensa de la Competencia de Andalucía, del Consejo de Defensa de la Competencia de Andalucía, por la que se aprueban los criterios para determinar la incidencia de un proyecto normativo en la competencia efectiva, unidad de mercado y actividades económicas. Boletín Oficial de la Junta de Andalucía, 13 de mayo de 2016, num. 90, pp.8-14.

Resolución de 30 de mayo de 2018, de la Dirección de la Agencia Andaluza de Instituciones Culturales por la que se convocan para el año 2018 las subvenciones reguladas por Orden de 19 de mayo de 2017, por la que se establecen las bases reguladoras de concesión de subvenciones, en régimen de concurrencia competitiva, a la producción de documentales en Andalucía. Boletín Oficial de la Junta de Andalucía, 7 de junio de 2018, num. 109, pp. 54-72.

Resolución de 30 de mayo de 2018, de la Dirección de la Agencia Andaluza de Instituciones Culturales, por la que se convocan para el año 2018 las subvenciones para la promoción del teatro, la música, la danza y el circo en Andalucía. Boletín Oficial de la Junta de Andalucía, 7 de junio de 2018, num. 109, pp. 73-187.

Resolución de 30 de mayo de 2018, de la Dirección de la Agencia Andaluza de Instituciones Culturales, por la que se convocan para el año 2018 las subvenciones para la promoción del tejido profesional del flamenco en Andalucía. Boletín Oficial de la Junta de Andalucía, 7 de junio de 2018, num. 109, pp.188-220.

Resolución de 30 de mayo de 2018, de la Agencia Andaluza de Instituciones Culturales, por la que se convocan para el año 2018 las subvenciones reguladas por la Orden de 1 de agosto de 2016, por la que se establecen las bases reguladoras de concesión de subvenciones, en régimen de concurrencia competitiva, a la producción de largometrajes en Andalucía. Boletín Oficial de la Junta de Andalucía, 8 de junio de 2018, num. 110, pp. 12-34.

Resolución de 21 de noviembre de 2018, de la Dirección General de Innovación Cultural y del Libro, por la que se convocan para el año 2018 las ayudas previstas en la Orden de 15 de octubre de 2018, por la que se aprueban las bases reguladoras para la concesión de subvenciones en régimen de concurrencia competitiva, de apoyo a las microempresas, pequeñas y medianas empresas culturales y creativas, para el fomento de su competitividad, modernización e internacionalización. Boletín Oficial de la Junta de Andalucía, 29 de noviembre de 2018, núm. 231, pp. 20-49.

Resolución de 28 de diciembre de 2018, de la Dirección de la Agencia Andaluza de Instituciones Culturales, por la que se amplía el plazo máximo de resolución y publicación del procedimiento de concesión de subvenciones en régimen de concurrencia competitiva, para la promoción del tejido profesional del flamenco en Andalucía, convocadas mediante Resolución de 30 de mayo de 2018 (BOJA núm. 109, de 7 de junio de 2018). Boletín Oficial de la Junta de Andalucía, 21 de enero de 2019, núm.13, pp. 12-13.

Román, M., \& Riscos, J. (2019). La mejora de la regulación económica en la Comunidad Autónoma de Andalucía. Revista de Economía ICE (907), marzo-abril, 115-130. Ministerio de Comercio, Industria y Turismo, Madrid. 